INPLASY

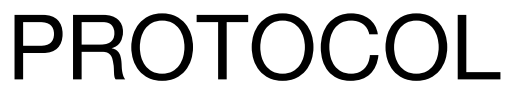

To cite: Yan et al. Effectiveness and safety of three different traditional Chinese therapies for asthma in minors: A systematic review and network meta-analysis protocol. Inplasy protocol 202090052. doi: 10.37766/inplasy2020.9.0052

Received: 11 September 2020

Published: 12 September 2020

Corresponding author:

ShiFan Yan

1539237955@qq.com

Author Affiliation: Jiangxi University of Traditional Chinese Medicine, Nanchang, China

Support: Grant number: 81704052.

Review Stage at time of this submission: The review has not yet started.

Conflicts of interest:

None.

\section{Effectiveness and safety of three different traditional Chinese therapies for asthma in minors: A systematic review and network meta-analysis protocol}

Yan, SF1; Yu, T2; Wang, $\mathrm{MH}^{3}$.

Review question / Objective: Traditional Chinese medicine (TCM) has been widely used in the treatment of asthma, but currently there has been no comprehensive evaluation on the efficacy and safety of different TCM therapies, based on network meta analysis(NET).

Condition being studied: The included articles determine the credibility of the final results. Therefore, we only select RCTS of traditional Chinese medicine therapy for asthma, and the intervention measures are only limited to acupuncture, moxibustion, acupoint sticking or blank control, excluding Chinese herbal medicine, massage, school and other traditional Chinese medicine therapies.

INPLASY registration number: This protocol was registered with the International Platform of Registered Systematic Review and Meta-Analysis Protocols (INPLASY) on 12 September 2020 and was last updated on 12 September 2020 (registration number INPLASY202090052).

\section{INTRODUCTION}

Review question / Objective: Traditional Chinese medicine (TCM) has been widely used in the treatment of asthma, but currently there has been no comprehensive evaluation on the efficacy and safety of different TCM therapies, based on network meta analysis(NET).

Condition being studied: The included articles determine the credibility of the final 
results. Therefore, we only select RCTS of traditional Chinese medicine therapy for asthma, and the intervention measures are only limited to acupuncture, moxibustion, acupoint sticking or blank control, excluding Chinese herbal medicine, massage, school and other traditional Chinese medicine therapies.

\section{METHODS}

Participant or population: For all minors (5-18 years old) diagnosed with Asthma, the diagnostic criteria follow Guidelines for the Diagnosis and Management of Asthma (EPR-3)13, and there are no requirements for country, region, race or gender.

Intervention: The intervention measures were only included in acupuncture, moxibustion and acupoint sticking. Among them, acupuncture tools could only choose millineedle, but there was no requirement for acupuncture needle application. Different moxibustion techniques were selected, including ginger moxibustion, heat-sensitive moxibustion, wheat grain moxibustion and mild moxibustion. There is no restriction on the use of Chinese herbs in acupoint application.Trials in multiple trial groups will also be included if all three of the above interventions are included.

Comparator: The intervention measures were only included in acupuncture, moxibustion and acupoint sticking. Among them, acupuncture tools could only choose millineedle, but there was no requirement for acupuncture needle application. Different moxibustion techniques were selected, including ginger moxibustion, heat-sensitive moxibustion, wheat grain moxibustion and mild moxibustion. There is no restriction on the use of Chinese herbs in acupoint application.Trials in multiple trial groups will also be included if all three of the above interventions are included.

Study designs to be included: The included articles determine the credibility of the final results. Therefore, we only select RCTS of traditional Chinese medicine therapy for asthma, and the intervention measures are only limited to acupuncture, moxibustion, acupoint sticking or blank control, excluding Chinese herbal medicine, massage, school and other traditional Chinese medicine therapies.

Eligibility criteria: 2.3.1 Types of studies The included articles determine the credibility of the final results. Therefore, we only select RCTS of traditional Chinese medicine therapy for asthma, and the intervention measures are only limited to acupuncture, moxibustion, acupoint sticking or blank control, excluding Chinese herbal medicine, massage, school and other traditional Chinese medicine therapies. 2.3.2 Type of participant For all minors (5-18 years old) diagnosed with Asthma, the diagnostic criteria follow Guidelines for the Diagnosis and Management of Asthma (EPR-3)13, and there are no requirements for country, region, race or gender. 2.3.3 Interventions The intervention measures were only included in acupuncture, moxibustion and acupoint sticking. Among them, acupuncture tools could only choose millineedle, but there was no requirement for acupuncture needle application. Different moxibustion techniques were selected, including ginger moxibustion, heat-sensitive moxibustion, wheat grain moxibustion and mild moxibustion. There is no restriction on the use of Chinese herbs in acupoint application. Trials in multiple trial groups will also be included if all three of the above interventions are included.

Information sources: Three English databases of PubMed, Cochrane Library, Embase and two Chinese databases of CNKI and Wanfang.

Main outcome(s): Spirometry: This can help us better assess the condition of the lungs, we use four main indicators: Forced vital capacity (FVC), forced expiratory volume in 1 s (FEV1), the FEV1/FVC ratio, and peak expiratory flow (PEF).

Additional outcome(s): 1) Asthma Quality of Life Questionnaire: It mainly includes five aspects: activity restriction, asthma symptoms, psychological status, response 
to stimuli, and concern for one's own health. There are a total of 35 questions, with a score of 1-7 for each question. Higher scores mean better results 2) The incidence rate of adverse events.

Quality assessment / Risk of bias analysis: The two authors (YT and YSF) evaluated the article methodology of inclusive trials independently, by the Cochrane collaboration "Bias risk" tool sequences generated from six aspects of allocation concealment, blind (or mask), incomplete data evaluation, evaluation reports and other sources of bias selective results. Finally, for each items, we made ranking of "Low-risk bias", "High-risk bias" and "Unclear" based on the Cochrane collaboration "bias risk" tool.

Strategy of data synthesis: If data is insufficient from the selected study, we will contact the author via email for the required data. If baseline and outcome data or other data are included, the mean and standard deviation of the change will be manually calculated according to the Cochrane.

Subgroup analysis: If the analysis shows significant heterogeneity, then the root cause will be analyzed according to the PICOS principle, and the STATA 14.0 will be used for subgroup analysis.

Sensibility analysis: We will evaluate the robustness of the meta-analysis results through sensitivity analysis, and exclude such as small-sample trials and low-quality trials to explore the impact of trial quality on efficacy estimates. In addition, we will conduct a second meta-analysis based on the results of the sensitivity analysis, summarize in tables and discuss.

Language: None restriction.

Country(ies) involved: China.

Keywords: asthma, network meta-analysis, protocol.
Contributions of each author:

Author 1 - ShiFan Yan - completed the project, search strategy, research selection, bias risk assessment, data extraction, data analysis and evidence quality assessment.

Author 2 - Ting Yu - completed the project, search strategy, research selection, bias risk assessment, data extraction, data analysis and evidence quality assessment. Author 3 - MaoHong Wang - conceived this study. 